Methods Retrospective analysis of case notes of children presenting to the PEC (Paediatrician with expertise in cardiology) clinic following diagnosis of secundum ASD in the neonatal period between 2014 and 2019.

Results 153 cases of secundum ASDs were identified of which 133 were considered to be appropriate and 20 discarded. Of these 20 cases, five were lost to follow up, 5 were diagnosed to be PFO (Patent Foramen Ovale) and 10 had other defects or were not diagnosed in the neonatal age group.

Of the 133 cases: 93 were $<3 \mathrm{~mm}, 23$ were between 3-5 $\mathrm{mm}$ and 17 were more than $5 \mathrm{~mm}$ on $2 \mathrm{D}$ measurement on echocardiography at diagnosis.

The cases were roughly followed up every 6 monthly intervals in the PEC clinic. The closure rate noted were as follows:

\begin{tabular}{llll}
\hline Size of ASDs & $<3 \mathrm{~mm}$ & $3-5 \mathrm{~mm}$ & $>5 \mathrm{~mm}$ \\
\hline Closed at 6 month f/u & $41(45 \%)$ & 0 & 0 \\
Closed at 1 year f/u & $45(48 \%)$ & $12(52 \%)$ & 0 \\
Closed at 2 year f/u & $7(7 \%)$ & $9(40 \%)$ & $8(47 \%)$ \\
Still Open & 0 & $2(8 \%)$ & $2(11 \%)$ \\
Needed surgery & 0 & 0 & $7(41 \%)$ \\
\hline
\end{tabular}

Discussion Secundum ASDs are often a common finding in neonatal echocardiography. Our study showed that the majority of these were small defects $(<5 \mathrm{~mm})$, none of which needed surgery. Roughly $85 \%$ of these closed by 1 year and the rest closed by 2 years of age. Of the ASDs more than 5 $\mathrm{mm}$ in size, around half of them closed spontaneously by 2 years of age and the other half needed surgery. This study helps to inform clinicians during consultations with parents and in deciding in regards to the frequency of follow ups.

\section{G12 EVALUATION OF PAEDIATRIC CARDIOLOGY SERVICES IN LOCAL CHILDREN'S CARDIAC CENTRES (LEVEL 3) IN THE UNITED KINGDOM}

${ }^{1} \mathrm{M}$ Graydon, ${ }^{2} \mathrm{~K}$ Bradly Russell, ${ }^{1,2} \mathrm{Y}$ Singh. ${ }^{1}$ School of Clinical Medicine, University of Cambridge, Cambridge, UK; ${ }^{2}$ Cambridge University Hospitals NHS FT, Cambridge, UK

\subsection{6/archdischild-2020-rcpch.4}

Background The NHS 'Safe and Sustainable' programme for children's congenital heart services in the UK has put significant emphasis on providing high quality services near home whenever possible, and Local Children's Cardiac Centres remain at the heart of this model. Data on the services provided by these centres have not been collected since 2014 and consequently their current practice is unknown.

Aims To evaluate the paediatric cardiology services currently provided by Local Children's Cardiac Centres in the UK, specifically looking at:

- The availability of Paediatricians with Expertise in Cardiology (PECs)

- The frequency of PEC-led clinics, and of outreach clinics led by a visiting paediatric cardiologist from a specialist centre

Methods An online survey was sent to members of the Paediatricians with Expertise in Cardiology Special Interest Group (PECSIG) and to contacts obtained via paediatric secretaries at Local Children's Cardiac Centres. Responses from hospitals that were not in the UK and from level 1 or 2 centres were excluded.

Results 92 responding hospitals met the inclusion criteria. The majority of these $(92 \%, 85 / 92)$ had at least one employed PEC. Multiple PECs were present at 42\% (39/92) of hospitals.

Of the 85 hospitals with PEC availability, 98\% (83/85) held regular PEC-led clinics. Most $(78 \%, 66 / 85)$ had 1 or more per week. $1 \%(1 / 85)$ held fewer than 1 per month and $2 \%(2 / 85)$ held no such clinics.

Outreach clinics led by paediatric cardiologists from specialist centres took place in 92\% (85/92) of the responding hospitals. In $85 \%(72 / 85)$ of these hospitals, these clinics occurred at least once per month on average. In the remaining hospitals with outreach clinics, frequency ranged from 4 to 10 times per year. $8 \%$ (7/92) had no outreach clinics.

Conclusion Most surveyed hospitals have established level 3 paediatric cardiology services provided by PECs, who lead local cardiac clinics. Over $90 \%$ of the hospitals have outreach clinics led by paediatric cardiologists from specialist centres. However, there remains a significant variation in the clinical services provided across different Local Children's Cardiac Centres.

\section{G13 TILT POSITIVE POTS (POSTURAL ORTHOSTATIC TACHYCARDIA SYNDROME): A CASE SERIES STUDY}

A Naz, S Johal, P Nair. Paediatric Department, Bedford Hospital NHS Trust, Bedford, UK

\subsection{6/archdischild-2020-rcpch.5}

Introduction PoTS (postural orthostatic tachycardia syndrome) is a condition of autonomic instability which causes significant tachycardia response on upright posture. It is also associated with a plethora of symptoms which can be very disabling for the child. A tilt positive PoTS is defined as a rise of heart rate more than $40 \mathrm{bpm}$ (in the 12-19 year age group) within the first 12 minutes (criteria 1) or a sustained heart rate of more than 120 beats per minute in the first 10 minutes (Criteria 2) with no hypotension or other reasons for tachycardia. Aim To do a retrospective analysis of 20 cases presenting to the PEC (Paediatrician with expertise in cardiology) clinic with positive diagnosis of PoTS on the head up tilt test (HUTT).

Method We did a retrospective analysis of children diagnosed with tilt positive PoTS, reviewing their clinical characteristics, symptomatology, results of tilt tests, treatment and outcome. Results Of the 20 cases, there were 5 boys and 15 girls with an average age of presentation at 12.7 years. $75 \%$ of the cases presented around 12-15 years of age. The predominant symptoms were as follows:

\begin{tabular}{ll}
\hline Symptoms & Percentage \\
\hline Dizziness & $100 \%$ \\
Headaches & $90 \%$ \\
Syncope & $55 \%$ \\
Abdominal Pain and Nausea & $45 \%$ \\
Palpitations & $35 \%$ \\
Chest Pain & $10 \%$ \\
Difficulty in breathing & $10 \%$ \\
Low Mood & $15 \%$ \\
Fatigue & $35 \%$ \\
\hline
\end{tabular}


4 of the patients also had a diagnosis of chronic fatigue syndrome (CFS). All patients had tilt tests (HUTT) of which fifteen satisfied criteria 1 and five satisfied criteria 2. The following medications were used:

\begin{tabular}{ll}
\hline Medications & Percentage \\
\hline Slow release sodium chloride & $75 \%$ \\
Beta Blockers & $45 \%$ \\
Fludrocortisone & $55 \%$ \\
Midodrine & $40 \%$ \\
Ivabradine & $20 \%$ \\
Others & $35 \%$ \\
\hline
\end{tabular}

Outcome after medications:

\begin{tabular}{ll}
\hline Outcome & Percentage \\
\hline Significant Improvement & $45 \%$ \\
Moderate Improvement & $45 \%$ \\
No/Mild improvement & $10 \%$ \\
\hline
\end{tabular}

Discussion PoTS is a multisystemic condition presenting typically in the adolescent girls with not many published studies in children. A tilt test helps to confirm the diagnosis and tailored management. Medications helps in symptomatic improvement in the majority with ability to resume education \& activities. Children with deconditioning type of PoTS secondary to CFS often show poor response to medications.

\section{G16(P) CHEST PAIN IN CHILDREN: DOES IT NEED CARDIOLOGY INPUT?}

B Sarder, S Johal, P Nair. Paediatric Department, Bedford Hospital NHS Trust, Bedford, UK

\subsection{6/archdischild-2020-rcpch.6}

Introduction Chest pain in children is a common complaint $\&$ is one of the commonest reasons for referral to a paediatrician with cardiology expertise (PEC) after heart murmurs. Although it causes high levels of anxiety in parents, the causes for these are usually benign. Often these children are referred to PECs for investigation and management.

Aim To look at the demographics of children presenting to the PEC outpatient clinic, review the presenting symptoms, investigations done and diagnosis with outcome.

Methods We reviewed the notes for the patient attending PEC clinic for complaints of chest pain over the last 3 years.

Results There were 40 referrals to the PEC clinic for children with chest pain. The age ranges were between 5-16 years with the average age being 13.8 years, with $55 \%$ being in the 12-16 years range. There were 17 boys and 23 girls. 29 children complained of bilateral, 8 left sided and 3 had central chest pain. 24 of these children had other symptoms like palpitations, dizziness, chest tightness, lethargy, chronic fatigue, breathing difficulties, anxiety, pain in the arms and fingers. All of them had further investigations that included blood tests, ECG, CXR, and echocardiography. Amongst the 26 children who had vitamin D levels done, 38\% had low levels, $42 \%$ had insufficient levels and $20 \%$ had normal levels. All patients had ECGs and echocardiography done which did not reveal any underlying cardiac cause for the chest pain. High dose vitamin D supplements were given for 9 cases. None of the cases needed cardiac interventions or treatment.

Discussion Chest pain is a common problem in adolescent children particularly in the 12-16 age group. Investigations were usually all normal, apart from findings of low vitamin D level in $80 \%$ of the children screened. Notably cardiac investigations were all normal suggesting that the possibility of underlying cardiac problems is very rare. Based on this study we recommend that chest pain in children should be seen in the general paediatric clinic and necessitates cardiac referrals or investigation only in a handful of these.

\section{G17(P) KAWASAKI DISEASE IN A 10 WEEKS OLD INFANT WITH CORONARY DILATATION}

MS Iqbal, AP Singh, E Haworth, S Raina. Department of Paediatrics, The Princess Alexandra Hospital, Harlow, UK

\subsection{6/archdischild-2020-rcpch.7}

10 weeks old infant presented to emergency with 9 days history of fever. He was seen by G.P twice during this illness, initially the impression of viral illness was made and on second time child had 9 days of fever and looked miserable and lethargic so was referred to padiatrics emergency.

There was no significant past medical history. No history of sick contacts and no recent travel. At presentation the child was febrile and irritable with no obvious source for fever. He was started on IV Ceftriaxone and Aciclovir. Initial bloods revealed profound leucocytosis (Total WBC 76, neutrophils 50.1) and CRP of 236, with serial blood films showing a reactive picture and left shift. Chest $\mathrm{x}$-ray was clear, urine/ blood cultures were negative and LP showed 8 WCC with negative cultures. After 72 hours of inpatient care the child was referred to tertiary centre in view persistent fever and irritability.

In the tertiary Hospital, parents told that child had bloodshot eyes, puffy swollen feet and red lips. Child was started on IVIG $2 \mathrm{gm} / \mathrm{kg}$ and high dose aspirin. Child had echocardiography which showed right coronary artery (RCA) dilatation maximal at distal segment measuring $3.58 \mathrm{~mm}$ with $\mathrm{Z}$ score of 8.86. RCA at mid-point and proximal segment measured 3.06 and 2.93 respectively with Z-score of 6.31 and 5.14 respectively. Left anterior descending artery measured 2.42 with Z-score of 4.27 .

During in-patient child was having on-going mild fever and in view of significant coronary dilatation child was started on low molecular weight heparin and also second line treatment with infliximab to which he responded well. Repeat echocardiography showed stable coronary dilatation.

Kawasaki is a mucocutaneous lymph node syndrome with specific diagnostic features, having said that it seems to be a rarity to find all the features consistent with diagnosis. The age presentation, less than 6 month is a very uncommon reported finding in UK and the lack of any diagnostic features initially for Kawasaki except fever lasting for more than 5 days makes this case an important academic and learning standpoint for paediatric trainees. 